\title{
Trajectory Planning for Reentry Maneuverable Ballistic Missiles
}

\author{
XIE Yu ${ }^{1, a^{*}}$, PAN Liang ${ }^{1, b}$ and YUAN Tianbao ${ }^{2, c}$ \\ ${ }^{1}$ College of mechatronic engineering and automation, \\ National university of defense technology, Changsha, Hunan, P.R.China \\ ${ }^{2}$ Equipment Academy of the Second Artillery Force, Beijing, P.R.China \\ a15111155218@139.com, bpanliang.2000@gmail.com, 'YLGW1977@126.com
}

Keywords: ballistic missile; trajectory planning; reentry maneuverable; Netown iteration; optimal control method.

Abstract. A trajectory planning scheme is proposed to satisfy the requirements of maneuver and terminal guidance constraints in the reentry flight for ballistic missiles. The reentry interface circle with the target point as its center is determined according to the range of the reentry phase. Then, a reference reentry point on the reentry interface circle is obtained as the terminal for the boost and unpowered trajectory planning, and the Newton iteration algorithm is employed to design the corresponding flight program. The reentry trajectory is divided into the pull-up, transition, horizontal flight and reentry phases. The optimal control and feedback control methods are used to deduce the reentry guidance law for each phase according to their trajectory characteristics. Simulations demonstrate that the generated trajectories can satisfy the requirements of maneuver, terminal guidance constraints and terminal precision.

\section{Introduction}

Typical ballistic missiles are inertial reentry without maneuver. In order to improve the maneuverability and terminal precision, the maneuverable reentry warheads are employed for some new ballistic missiles. The intermediate-range ballistic missile "Pershing II" is one of the typical maneuverable reentry missiles [1]. In the reentry flight, the missile can pull up by aerodynamic force, and decrease in velocity to complete the radar image matching, then dive to attack the target with an nearly 90 degree falling angle. The range is over $1800 \mathrm{~km}$, and the precision is less than $30 \mathrm{~m}$. However, there is no more complete details about the trajectory planning and guidance algorithm for "Pershing II" missile in public.

Many reentry maneuverable trajectory planning methods have been developed to improve the penetrability of ballistic missiles in their reentry flight. Che et al. [2] employed the genetic algorithms to generate the optimal trajectory for maneuverable warhead; References. [3-5] obtained the optimal reentry maneuver trajectories based on optimal control theory and numerical analysis; The reentry spiral maneuver trajectory generation approach was studied in [6]. Based on these works, this paper proposes a reentry maneuverable trajectory planning scheme considering the requirements of improving penetrability and satisfying the terminal radar guidance constraints in reentry flight. Moreover, several times of horizontal flight at different altitude in reentry is studied to satisfy more complex reentry flight mission.

\section{Analysis of Trajectory Characteristics}

The "Pershing II" missile is employed as an example to analyze the reentry maneuverable trajectory characteristics. As shown in Fig.1, the typical flight course consists of the boost, unpowered and reentry phases. 


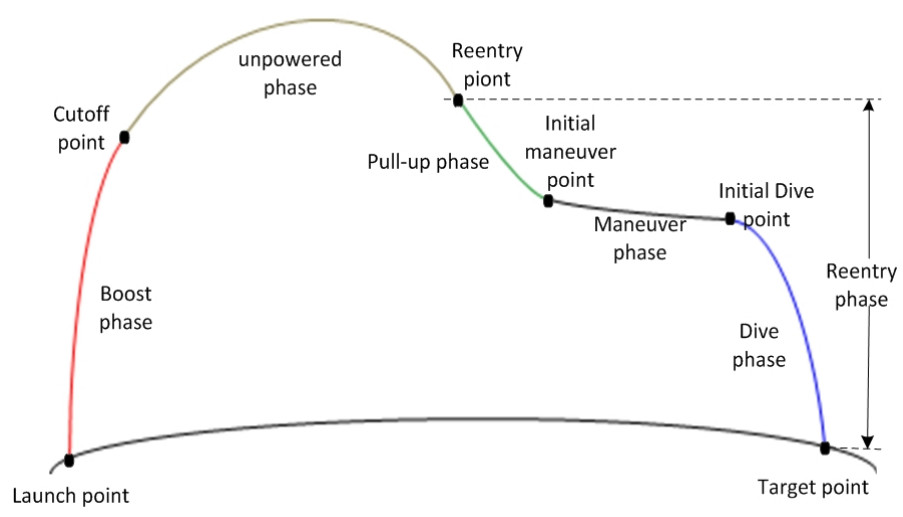

Figure 1 Typical flight course of "Pershing II" missile

In the boost phase, the missile takes off vertically and turns gradually toward the target. The unpowered flight is in the nearly vacuum space, and the gravity is the only external force. Hence, the terminal states in this phase is determined by the initial states, namely the terminal states of the boost phase. Hence, only the flight program of the boost phase should be designed to satisfy the state constraints at the reentry point. The reentry phase can be divided into the pull-up, maneuver and dive phases. The trajectory characteristics in each phase is quite different. Hence, different guidance laws are developed to generate the corresponding trajectories.

\section{Planning Algorithm}

Algorithm Description. Figure 2 shows the flowchart of the reentry maneuverable trajectory planning algorithm.

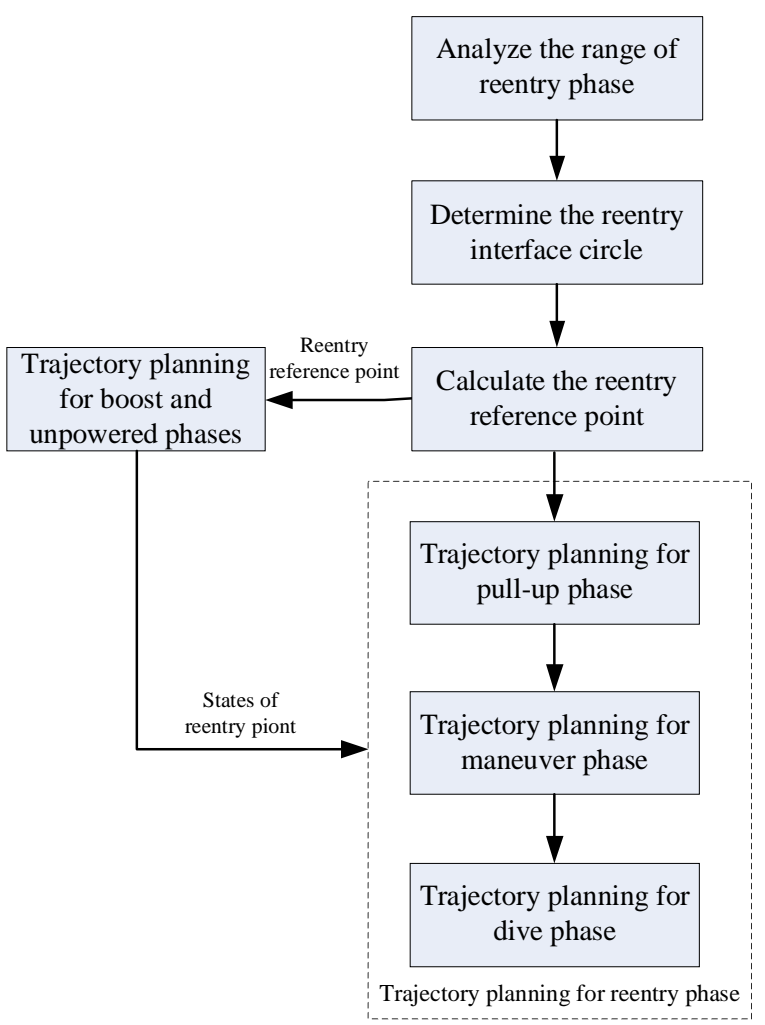

Figure 2 Trajectory planning algorithm

The steps for the algorithm are as follows:

Step 1: analyze the characteristics of reentry maneuver, determine the range to fly in the reentry phase. 
Step 2: obtain the reentry interface circle with the target point as its center (as shown in Fig. 3); determine the reference reentry points on the reentry interface circle as the terminal for the boost and unpowered trajectory planning.

Step 3: plan the boost and unpowered trajectories to satisfy the state variables at the reference reentry point. The terminal states and control variables are used as the initial conditions for the trajectory planning of the reentry phase.

Step 4: plan the pull-up, maneuver, and dive trajectories respectively to satisfy the maneuver, terminal radar guidance constraints, and terminal states.

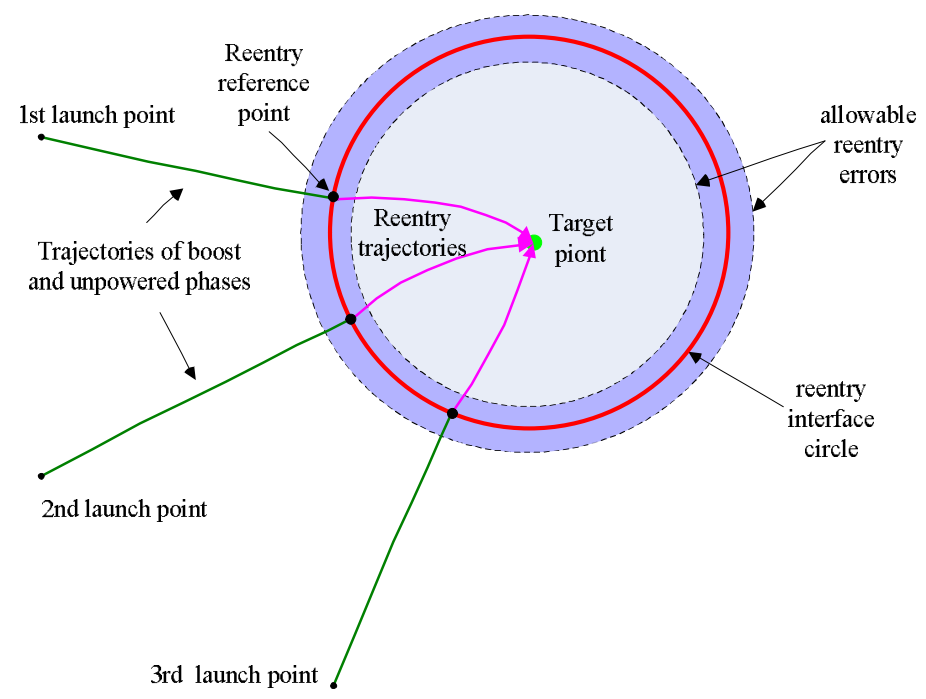

Figure 3 The reentry interface circle and trajectories

Planning Algorithm for Boost and Unpowered Phases. As mentioned before, only the key flight program, that is, the azimuth and the attitude angles of pitch, yaw, and roll, should be determined to generate the boost and unpowered trajectories. Both the yaw and roll angles are usually set to zero, and the pitch angle is parameterized and determined by several variables such as the absolute value of maximum negative angle of attack, pitch angular velocity. In this paper, the azimuth angle $A_{0}$ and the pitch angular velocity $\boldsymbol{\alpha}_{p_{2}}$ of the second stage are chosen as the variables to plan the boost and unpowered trajectories.

The purpose of flight program calculation is to reduce the position error at the reentry point, which is given by

$$
\begin{aligned}
& \Delta L_{r}=\Delta L_{r}\left(\psi_{\alpha_{2}}, A_{0}\right) \\
& \Delta H_{r}=\Delta H_{r}\left(\psi_{p 2}, A_{0}\right)
\end{aligned}
$$

where $\Delta L_{r}$ and $\Delta H_{r}$ are the downrange and cross-range at the reentry point, respectively.

The planning algorithm is shown in Fig.4, and it can be expressed as follows:

Step 1: initialize the variables: $\psi_{p_{2}}=\boldsymbol{Q}_{p_{2}}^{(0)}, A_{0}=A_{0}^{(0)}$, where $A_{0}^{(0)}$ is obtained by spherical triangle, and $\phi_{p_{2}}^{(0)}$ is a small preselected negative value according to experience.

Step 2: calculate the boost and unpowered trajectories, and determine the position errors of $\Delta L_{r}^{(0)}$ and $\Delta H_{r}^{(0)}$ at the reentry point. If $\Delta L_{r}^{(0)}$ and $\Delta H_{r}^{(0)}$ are small enough, stop the algorithm and calculate the corresponding trajectory using the generated variables ${\underset{p}{2}}_{2}^{0)}$ and $A_{0}^{(0)}$; otherwise, the algorithm proceeds to Step 3.

Step 3: calculate the partial derivative $\partial \Delta L / \partial A_{0}, \partial \Delta H / \partial A_{0}, \partial \Delta L / \partial \psi_{p_{2}}$, and $\partial \Delta H / \partial \psi_{p_{2}}$, respectively. Herein, the Richard method is employed to obtain the partial derivative for improving iteration precision as follows [7] 


$$
\left\{\begin{array}{l}
f_{x_{i}}{ }^{\prime}\left(x^{i}\right) \approx \frac{4}{3} \frac{\omega_{i}(h / 2)-\omega_{i}(-h / 2)}{h}-\frac{1}{6} \frac{\omega_{i}(h)-\omega_{i}(-h)}{h} \\
\omega_{i}(h)=f\left(x_{1}^{0}, \mathrm{~L}, x_{i}^{0}+h, \mathrm{~L}, x_{n}^{0}\right)
\end{array}\right.
$$

where $h$ is the iteration step. Obviously, we have to calculate trajectory for four times to obtain the partial derivative according to Eq. (2).

Step 4: update the values of variables as follows

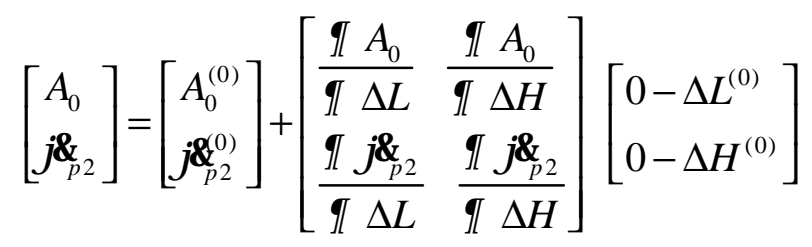

where the partial derivative matrix is given by

$$
\left[\begin{array}{lll}
\frac{\partial A_{0}}{\partial \Delta L} & \frac{\partial A_{0}}{\partial \Delta H} \\
\frac{\partial \psi_{p 2}}{\partial \Delta L} & \frac{\partial \psi_{p 2}}{\partial \Delta H}
\end{array}\right]=\left[\begin{array}{ll}
\frac{\partial \Delta L}{\partial A_{0}} & \frac{\partial \Delta L}{\partial \alpha_{p 2}} \\
\frac{\partial \Delta H}{\partial A_{0}} & \frac{\partial \Delta H}{\partial \psi_{p 2}}
\end{array}\right]^{-1}
$$

Let $A_{0}^{(0)}=A_{0}$ and $\oint_{p_{2}}^{(0)}=\psi_{p_{2}}$, and repeat steps 2-4 until the position error at the reentry point is small enough.

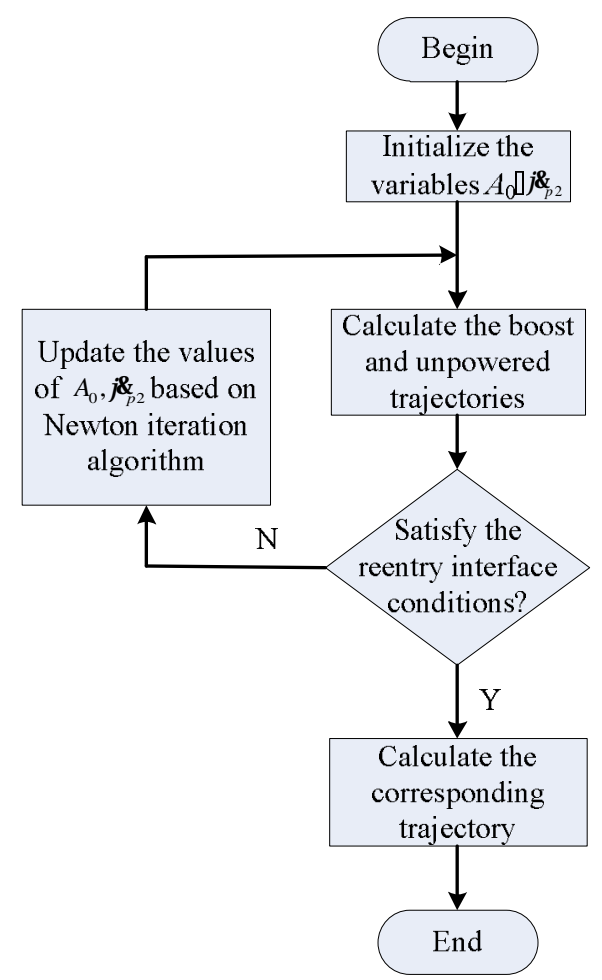

Figure 4 Flowchart of the boost and unpowered trajectory planning

Planning Algorithm for Reentry Phase. As shown in Fig. 5, assume that $O_{T}$ is the target point and $O_{1}$ is the warhead's body centroid. The relative motion equations [8] in the target frame can be expressed as

$$
\begin{aligned}
& \chi_{D}^{\alpha}=\left(\frac{1 \alpha}{v}-\frac{2 \beta \alpha}{\rho}\right) \lambda_{D}^{\alpha}-\frac{\rho \alpha}{\rho} \gamma \delta \\
& \alpha_{T T}^{\alpha}=\left(\frac{1 \alpha}{v}-\frac{2 \beta \alpha}{\rho}\right) \lambda_{T T}^{\alpha}+\frac{\rho \alpha}{\rho} \gamma{ }^{\prime}
\end{aligned}
$$


where $\lambda_{D}$ is the line-of-sight angle in dive plane, $\lambda_{T T}$ is the azimuth angle in bank plane, $v$ is the Earth-relative velocity magnitude, and $\rho$ is the distance between the warhead's body centroid and target point. $\gamma_{D}$ and $\gamma_{T}$ are azimuth angle of velocity vector in dive plane and bank plane, respectively.

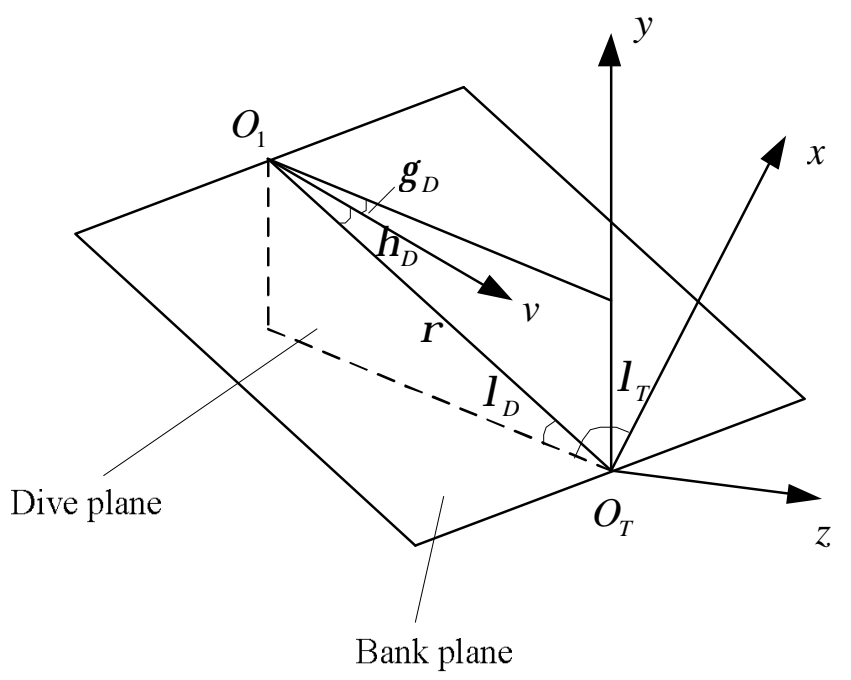

Figure 5 Dive and bank planes

The reentry phase consists of the pull-up phase, maneuver phase and dive phase. Different planning algorithms are developed according to the trajectory characteristics.

Pull-up phase. In this phase, the atmosphere is relative thin. The aerodynamic lift is produced on the action of the trim angle-of-attack to pull up the warhead gradually until the flight path angle $\theta_{T}$ approximate to zero. Suppose that both the bank angle and sideslip angle are zero, and the angle of attack is a constant value $\bar{\alpha}$ in the pull-up flight. Hence, the algorithm is to calculate the value of $\bar{\alpha}$ iteratively until $\theta_{T} \approx 0$ when the flight altitude is equal to the preselected value $\bar{h}$.

Horizontal flight phase. In order to satisfy the constraints of terminal guidance such as the radar image matching, both the flight altitude and heading should meet the preselected values in this phase. The longitudinal and lateral overloads are obtained by employed negative feedback as Eq.(6) to keep the warhead flying horizontally and toward the determined azimuth.

$$
\begin{aligned}
& N_{y}=1+k_{h}\left(h-h^{*}\right) \\
& N_{z}=v\left(k_{a} \lambda \frac{\alpha}{T}+k_{b} \frac{\rho \alpha}{\rho}\left(\sigma_{T}-\sigma_{T}^{*}\right)\right) / \mathrm{g}_{0},
\end{aligned}
$$

where $h$ and $\sigma_{T}$ are the flight altitude and heading angle, and the superscript "*" denotes the preselected values. $k_{h}, k_{a}$ and $k_{b}$ are feedback coefficients.

Suppose that the control pattern of warhead is slide-to-turn (STT). Then, the bank angle is zero, and the normal force coefficient $C_{y}$ and the lateral-normal force coefficient $C_{z}$ are given by

$$
\begin{aligned}
& C_{y}=\frac{N_{y} M g_{0}}{q S_{m}} \\
& C_{z}=\frac{N_{z} M g_{0}}{q S_{m}},
\end{aligned}
$$

where $M$ is the mass of the warhead, $g_{0}=9.81 \mathrm{~m} / \mathrm{s}^{2}, q$ is dynamic pressure, and $S_{m}$ is the reference area. The control variables of attack and sideslip angles can be determined by interpolating the aerodynamic table inversely. The magnitude constraints of control variables should be considered. 
Dive phase. The dive phase is the last flight phase. The main purpose of this phase is to arrive at the target point with the required falling angle, that is, the terminal variables including flight path angle and heading angle should be satisfied. Hence, the optimal guidance law is expressed as

$$
\begin{aligned}
& \varkappa \&=k_{1} \lambda_{D} \alpha k_{2} \frac{\beta \alpha}{\rho}\left(\lambda_{D}+\theta_{T}\right) \\
& \psi \alpha=k_{3} \lambda_{T} \alpha k_{4} \frac{\beta}{\rho}\left(\lambda_{T}+\sigma_{T}\right)
\end{aligned},
$$

where $k_{1}, k_{2}, k_{3}$ and $k_{4}$ are proportional coefficients; $\theta_{T}$ and $\sigma_{T}$ are the required terminal flight path angle and heading angle, respectively.

Hence, the Lateral and longitudinal overloads are given by

$$
\begin{aligned}
& N_{y}=v \delta / g_{0} \\
& N_{z}=v \lambda \phi / g_{0}
\end{aligned}
$$

The values of attack and sideslip angles can be obtained as mentioned in the horizontal flight phase. Transition phase. The transition phase is to complete the transition flight between two phases such as the phase between the pull-up and the horizontal flight phases. The guidance law is similar to the law of the dive phase as shown in Eq. (8), except the values of terminal flight path angle and heading angle.

\section{Simulations}

The "Pershing II" model obtained in public [1] is used in the simulations. The mission scenario is designed as follows: both the initial longitude and latitude at the launch point are set to zero. The target longitude and latitude are $15^{\circ}$ and $0^{\circ}$, respectively. The range of the reentry phase is $200 \mathrm{~km}$. In the course of reentry, two pull-up phases are required to complete the radar image matching or reconnaissance, and the flight altitudes are $20 \mathrm{~km}$ and $10 \mathrm{~km}$, respectively. The altitude where the warhead of "Pershing II" pulls up is set to $20 \mathrm{~km}$. The coefficients are set as follows: $k_{h}=-0.005$, $k_{a}=-4, k_{h}=-2, k_{1}=-4, k_{2}=-2, k_{3}=-4$, and $k_{4}=-2$.

As shown in Fig.6, the zoom-in view near the reentry phase is shown in the inset at the bottom of the figure. The range is $1667.928 \mathrm{~km}$. The warhead completes the predesigned pull-up flight, and then keep flying horizontally at the altitude of $20 \mathrm{~km}$ and $10 \mathrm{~km}$, respectively. Figure 7 shows the ground track of the generated trajectory. The position error at the target point is nearly equal to zero without considering the random error of aerodynamic coefficients and atmosphere model. There is a lateral maneuver in the ground track corresponding to reentry phase for satisfying the constraints of radar image matching in the preselected matching area. Figures 8 and 9 show the flight path angle and velocity vs range, respectively. The zoom-in view near the horizontal flight phases is shown in the inset at the top of Fig.8. The flight path angle in the horizontal flight phases is nearly equal to zero, which means the warhead keep flying horizontally successfully. The attitude angles and angles of attack, bank and sideslip are shown in Figs. 10 and 11, respectively. We notice that most of the angles change acutely to complete the complex reentry flight, which is quite different from the corresponding angles of typical inertial reentry missiles. 


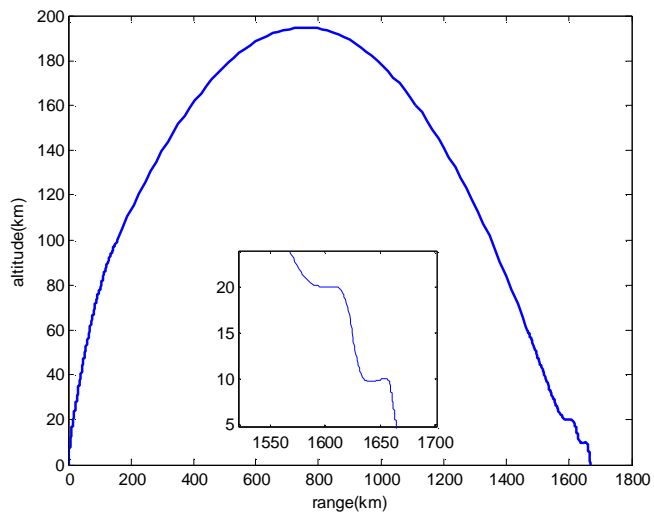

Figure 6 Altitude vs range

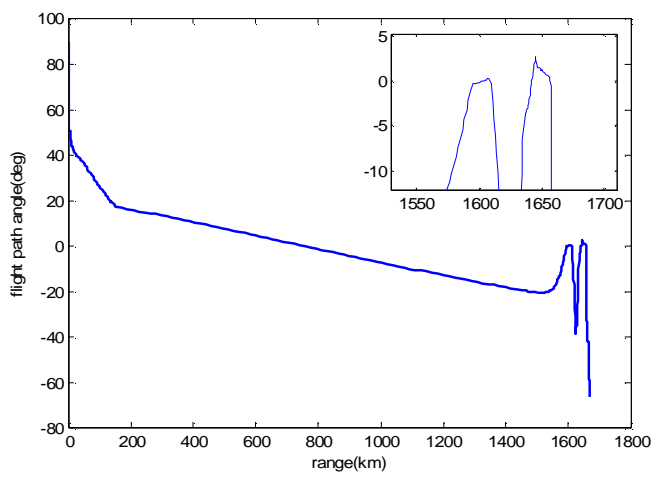

Figure 8 Flight path angle vs range
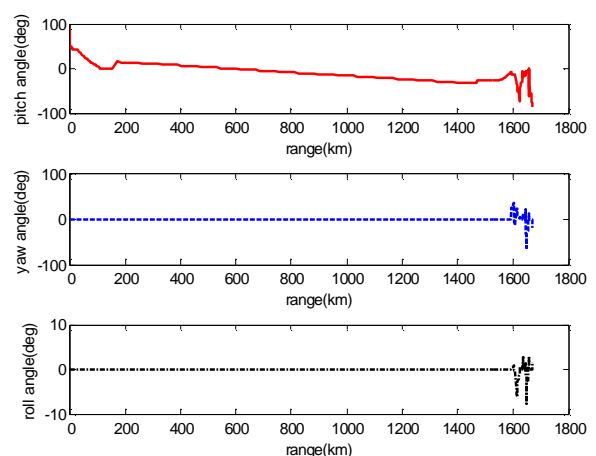

Figure 10 Attitude angles vs range

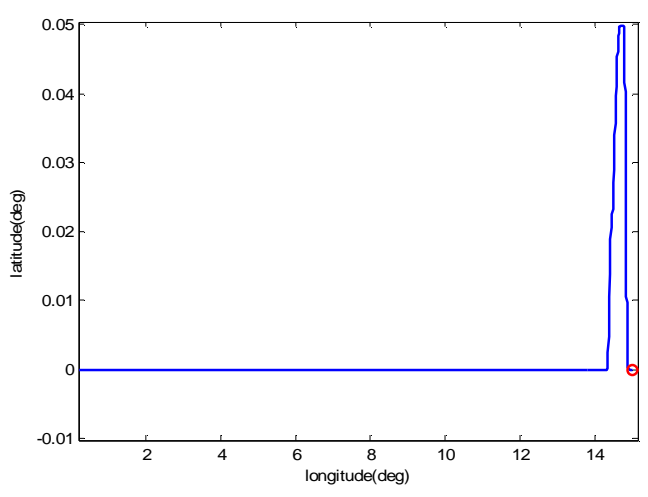

Figure 7 Latitude vs longitude

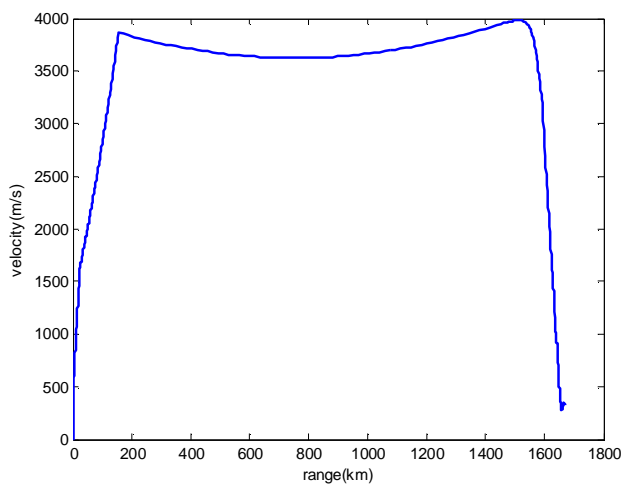

Figure 9 Velocity vs range
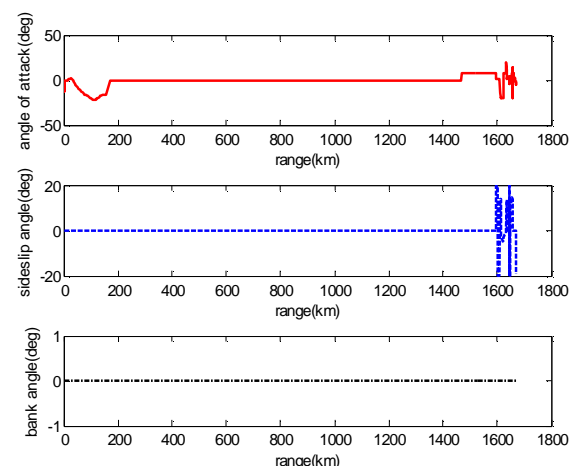

Figure 11 Angles of attack, bank and sideslip vs range

\section{Conclusions}

A reentry maneuverable trajectory planning scheme for ballistic missiles has been presented. The trajectory planning algorithm includes two main parts, that is, the trajectory planning of boost and unpowered phase and the reentry trajectory planning. The former is to design the key variables corresponding to flight program for satisfying the position error at the reentry point, and the latter is to satisfy the maneuver, terminal guidance constraints, and terminal precision at target point. The simulations based on "Pershing II" missile's model denote the planning algorithm is effective. Considering the space limitations, both the perturbation such as the atmosphere model and the flight constraints are not considered in the paper, which is left for future research. 


\section{Acknowledgements}

This work was financially supported by National Natural Science Foundation of China (11502289).

\section{References}

[1] P. Huang. Prelimiary analysis of Pershing II missile and its warhead, Missiles and Space Vehicles, vol. 207, 1994, pp. 7-15(in Chinese).

[2] J. Che, W Wang, K. He, Optimization design of reentry trajectory for maneuverable warhead based on genetic algorithms, Journal of Ballistics, vol. 21, Mar. 2009, pp. 43-46(in Chinese).

[3] H. Wang, X. Liu, Design of reentry maneuvering trajectory for ballistic missile, Computer Simulation, vol. 25, Jun. 2008, pp. 59-61(in Chinese).

[4] H. Zhao, Research on reentry maneuver trajectory design for ballistic missile, Journal of Astronautics, vol. 1, Apr. 1985, pp. 1-10(in Chinese).

[5] Y. Chen, Y. Zhang, Reentry maneuvering trajectory design of TBM on attacking natural defense target, Flight Dynamics, vol. 22, Sep. 2004, pp. 53-56(in Chinese).

[6] S. Li, X. Ren, R. Wu, Research of spiral maneuver of reentry missile, Journal of Astronautics, vol. 21, Oct. 2000, pp. 41-48(in Chinese).

[7] T. Li, Optimal calculation principle and program design, Changsha: Press of National University of Defense Technology, 2012(in Chinese).

[8] H. Zhao, Reentry dynamics and guidance for vehicles, Changsha, 1997(in Chinese). 